\title{
Construction Details of an Autoradiographic-Based Rat Brain Phantom for Emission Tomography
}

\author{
D.A. Dougherty, ${ }^{1}$ J. Nissanov ${ }^{2}$ G.R. Gindi ${ }^{3}$ \\ ${ }^{1}$ Department of Anatomy, SUNY Stony Brook \\ ${ }^{2}$ School of Biomedical Engineering, Drexel University \\ ${ }^{3}$ Department of Radiology, SUNY Stony Brook
}

\begin{abstract}
A digital brain phantom was created from rat brain autoradiographic (AR) data for use in emission computed tomography studies. The animal tissue was radio-labeled with $\left[{ }^{14} \mathrm{C}\right]$-2-deoxyglucose, a functional analog of the PET agent $\left[{ }^{18} \mathrm{~F}\right]$-fluoro-deoxyglucose. Following sacrifice of the animal, serial tissue sections were cut at $20 \mu \mathrm{m}$ thickness, digitized, and calibrated to represent the ground truth $2 \mathrm{D}$ relative spatial distribution of radionuclide within the tissue. A 3D representation was achieved by digital alignment of the serial AR images to corresponding video blockface images acquired at the time of cutting. In addition, a magnetic resonance data set was co-registered to the AR and blockface data using the AIR algorithm. This paper is concerned with outlining the details of construction for this phantom.
\end{abstract}

\section{INTRODUCTION}

We have been exploring the hypothesis that biologically realistic phantoms are useful for testing reconstruction strategies in nuclear medicine [1] [2]. To obtain a realistic spatial density of radionuclide, we use autoradiographic (AR) data from animal studies. This is because radiopharmaceuticals used for medical imaging can also expose autorad film (via $\beta$ emission), yielding high-resolution, low-noise 2-D records of the essentially ground-truth radionuclide density in thin tissue slices. In previous work [2], we utilized data from a rhesus monkey to obtain a human-like phantom, given the anatomical homology between the primate and human brain following a gross scale factor. In the current work, we describe construction of a rat brain phantom. Here, the motivation is that there is extensive use of the rat as an experimental subject in nuclear medicine testing using small animal PET and SPECT scanners, and that improved image quality in this venue is a worthwhile goal.

This work describes the acquisition stages and image processing tasks associated with the construction of a realistic, fully 3D, brain phantom from a normal adult male Sprague Dawley rat brain within the intact skull, as shown in Figure 1. Specific advantages of this data set include the presence of the skull for attenuation modeling and tissue integrity, complete AR data continuity allowing for 3D projection and reconstruction, blockface video imaging providing an alignment framework, and co-registered high-resolution MR for segmentation at the level of clinical resolution. Each of these aspects represents a significant advance from our previous studies. We have not yet applied the rat phantom to image quality evaluation goals, although in previous work
[2] with primate AR data we have shown that the results of phantom simulations for quantitation tasks are dependent upon the nature of the phantom itself.

The final phantom consists of 3D co-registered autoradiograph, tissue blockface images, and MR. The calibrated autoradiograph provides a record of relative radionuclide density from which photon generation and image formation have been simulated by a Monte-Carlo generator (SimSet). The blockface images provide a fixed anatomical coordinate frame for registering the autorad, but can also be used as an aid in segmentation of tissue components. The MR dataset is useful in various testing goals, for example as MR side information in reconstruction. There also exists the potential for combining a segmented blockface image with an MR image to form a validation set for MR segmentation algorithms, where the blockface would constitute a ground-truth and the MR would be a test set.

In the sections that follow, the multiple stages of animal preparation, tissue handling, and data processing are discussed in the order that they were performed.

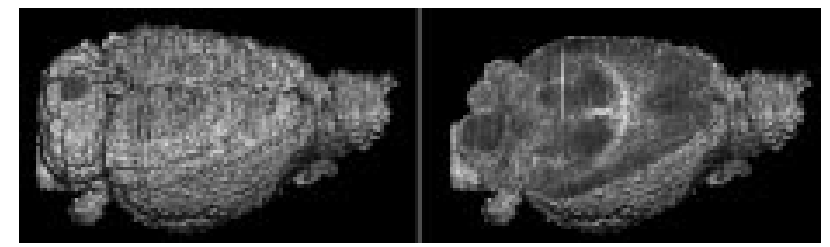

Figure 1: Shown is the autorad data of the 3-dimensional rat brain phantom in the intact skull. The image in the right panel demonstrates the interior structure of the brain in the horizontal plane (original plane of section was coronal).

\section{Methods}

\section{A. Magnetic Resonance Imaging (MRI)}

Prior to sacrifice, a multispectral MR study was performed on the subject. Here, multispectral MR includes spin-lattice $\left(\mathrm{T}_{1}\right)$, spin-spin $\left(\mathrm{T}_{2}\right)$, proton density $(\rho)$ acquisitions. MR is necessary for two major reasons. First, the MR is used in our work involving prior information for reconstruction. Here, the multispectral data is needed as input for a segmentation algorithm and the output of that operation is then used as side information for the reconstruction algorithm. Secondly, MR provides segmentation data at the level of clinical resolution, allowing for its use as a segmentation phantom. This is important because information from the proposed very realistic 
phantom will be compared to data that could be derived from current clinical techniques. While delineation of the internal structures of the brain can be achieved with far greater accuracy using histological material, this does not simulate clinical resolution. MR is, therefore, to be used as the model of clinical segmentation and associated errors.

MR acquisition was done using a 4.7Tesla Bruker Avance magnet. Pulse sequences were TR/TE of 1000/22, 2000/34, and 3300/60 for $\mathrm{T}_{1}, \mathrm{~T}_{2}$, and $\rho$, respectively. A homemade saddle coil was used, which fit over the animal head with a $1 \mathrm{~cm}$ tolerance. The field of view (FOV) was $4 \mathrm{~cm}$ with matrix size of 128 by 128 accounting for an inplane spatial resolution of approximately $300 \mu \mathrm{m}$. Slice thickness was $1 \mathrm{~mm}$. Since orthogonal acquisitions were collected in order to have the higher in plane resolution data available from the three major planes of section, a total of 16 horizontal, 22 sagittal, and 28 coronal slices were produced. Each individual acquisition lasted on the order of 20 minutes. An example MR image is shown in Figure 2, panel D.

During the MR acquisition, animals must remain motionless and therefore must be anesthetized. Induction of anesthesia is done with $5 \%$ halothane delivered through a cone that fits over the animal head. During the scan, a surgical level of anesthesia was maintained with a humidified, 1-2\% halothane gas mixture supplemented with 1 liter/min $\mathrm{O}_{2}$ and 1 liter/min $\mathrm{NO}_{2}$. Ventilation of the animal with this gas mixture was maintained by a Harvard Instruments (Harvard Instruments Co., South Natick, MA) model 683 rodent ventilator and a 14gauge intravenous catheter was used as the tracheal tube (stylus removed, inserted to a depth of $50 \mathrm{~mm}$, fixed in place with suture). The stroke volume was set to $2-3 \mathrm{cc}$, with a frequency of 60 breaths/min, designed to keep the blood parameters within the physiological range $\left(\mathrm{pH} 7.3-7.4, \mathrm{CO}_{2}\right.$ $35-40 \mathrm{mmHg}, \mathrm{O}_{2} 180-200 \mathrm{mmHg}$ ). Because the ventilator apparatus is largely constructed of ferrous materials, it was necessarily positioned outside of the MR scanner room. This location required use of a 3 meter long ventilation tube. Despite the apparent deadspace, arterial gas measurements and observation of conjunctiva and mucosa color indicated that the animal was, nonetheless, being adequately ventilated.

\section{B. 2DG Labeling}

The specific tracer chosen was $\left[{ }^{14} \mathrm{C}\right] 2$-deoxy-glucose (2DG), shown to be functionally equivalent to the ECT agent $\left[{ }^{18} \mathrm{~F}\right] 2$-fluoro-deoxy-glucose (FDG) by Som et al. [3]. In that experiment, the researchers prepared tissue that was double labeled with both 2DG and FDG. Due to the vast difference in the half life of each isotope, two film sets were able to be produced that had a normalized exposure contribution from both isotopes. Subsequent image analysis demonstrated the very close correspondence in brain uptake of both species. Although the correlation coefficients of regional brain uptake were on order of 0.9 , direct comparison of absolute accumulation was not performed because it was necessary to use separate calibration standards for each species.

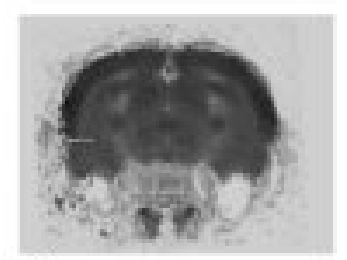

A.

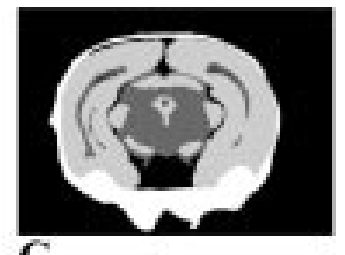

C.

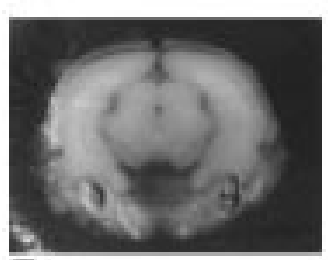

B.

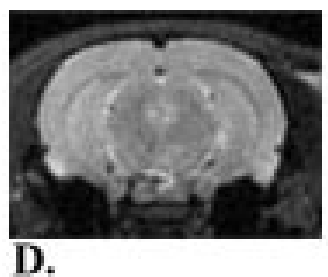

Figure 2: Corresponding slices of each of the following datasets are shown: A.) Autoradiographic image of the rat brain labeled with 2-DG; B.) Blockface video image of the remaining specimen immediately following collection of the previous AR slice; C.) Segmentation of the tissue slice into regions of bone (white), csf (black), gray (light gray) and white matter (dark gray); D.) Magnetic resonance image from the same animal prior to sacrifice.

While fully conscious, a bolus of $0.5 \mathrm{ml} 2 \mathrm{DG}$ (specific activity $0.1 \mathrm{mCi} / \mathrm{ml}$ ) was injected i.p. The animal was then returned to its cage for one hour prior to sacrifice. At the time of sacrifice, the animal was given a pentobarbital overdose $(0.5 \mathrm{ml}, 50 \mathrm{mg} / \mathrm{ml})$, producing death in under 10 minutes.

Following death, the animal was decapitated and the skin, musculature and mandible were removed. The brain was then quickly frozen within the skull, thus preventing mechanical distortions of the brain shape. The method used for freezing the brain can have dramatic consequences on gross distortion. If one freezes the brain in situ (as was done for the brain in Figure $3 \mathrm{~B}$ ), distortion is minimized from the in vivo state (as shown by the MR image in Figure $3 \mathrm{~A}$ ). In the present study, we cut the tissue while it was still encased in the skull. This does compromise section quality, as seen in Figure $2 \mathrm{~A}$. We have been investigating alternatives. Removal of the brain from the full skull after freezing and prior to cryosectioning is not feasible. While removal of the dorsal frontal, parietal and interparietal bone plates is easy, separation of the ventral brain surface from bone is quite difficult. If, however, one removes all but the dorsal skull prior to freezing, completion of the brain separation from the skull after freezing is easy and the tissue distortion is kept to a minimum (as was done for the brain in Figure $3 \mathrm{C}$ ). We refer to this freezing procedure as "skullcap" and it will be our method of choice for future work. This method is substantially better than a standard histological freezing procedure (Figure $3 \mathrm{D}$, procedure described in legend). For freezing, the specimen was placed on a platform and slowly lowered into the freezing medium of isopentane, chilled in a double dewar design with dry ice cooled methanol. Following 15 minutes in this medium the specimen is removed and placed into a (-80) degree Celsius freezer until time of sectioning. Due to the long $\mathrm{T}_{1 / 2}$ of $\left[{ }^{14} \mathrm{C}\right](5,730$ years), activity loss during this period is negligible. 

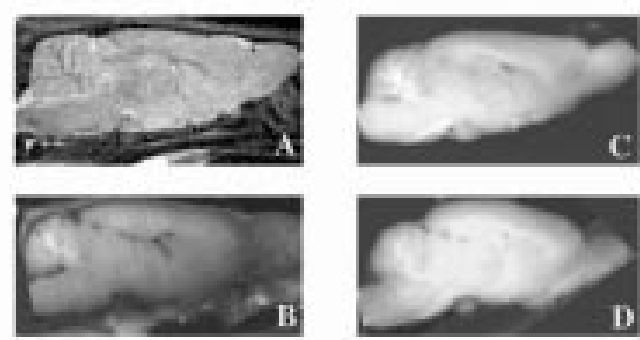

Figure 3: Freezing methods and distortion. Homologous views from 3 brains. A) In vivo state imaged by MR. B) Blockface image of the same brain as in A during cutting of the tissue while it is still encased in the skull. C) Blockface view of a brain prepared by the skullcap method. D) Blockface image of a brain frozen by suspension method. In the latter technique, the brain is completely removed from the skull and is suspended by the spinal cord and attached shoulder musculature. It is then lowered into cold isopentane.

\section{Histological Procedures}

A Leica Cryopolycut microtome was used to section the tissue using a tungsten carbide blade. The specimen is removed from the freezer and fixed to the microtome chuck in a 10:1 mixture of Lipshaw M-1 embedding medium with India ink added to enhance figure-to-ground contrast in the blockface image. Alignment of the specimen within the microtome chuck was performed by eye. For sectioning, every fifth coronal slice of the brain was taken at $20 \mu \mathrm{m}$ thickness throughout the rostro caudal axis, during which time the microtome chamber was maintained at $(-20)^{\circ} \mathrm{C}$. An important consideration in accurate 3D reconstruction of histological material is the accuracy of the advance mechanism of the microtome. We have previously developed a method to assess cutting thickness [4]. The calibration utilizes a contact profilometer to measure polyvinyl chloride (PVC) cut on the instrument. The material used closely matches the hardness of brain and has low thermal expansion coefficient. We have used this method to evaluate the Leica Cryopolycut used for this study. When set to advance $20 \mu \mathrm{m}$, our average section thickness was $19.26 \pm 1.678 \mu \mathrm{m}$. The thickness oscillates with a period of 2 sections.

Tissue cutting typically distorts the sections, and tape systems to minimize this are commercially available. We have examined the performance of two of these systems, the 3M tesafilm 4248 and the Instrumedics TW system. The former is simply a tape that is placed over the blockface and supports it during cutting. The section remains on the tape. In the latter system, a tape is again used, but the section is transferred to a polymer-coated slide and UV irradiation cures the polymer, thus adhering the section to the glass slide. The tape is then stripped off. While both are effective in limiting distortion to less than $60 \mu \mathrm{m}$ [Nissanov, Bertran and Tretiak, unpublished findings], the latter supports a broader range of histological procedures. While the $3 \mathrm{M}$ tape is usable with autoradiography, and, indeed we used it in the present study, sections collected on it cannot be subsequently stained using procedures requiring organic clearing agents. An example AR slice is shown in Figure 2, panel A. We note that the quality of the autoradiogram is sub-optimal as a consequence of brain freezing within the skull.

\section{Blockface Imaging}

The microtome is a large horizontal system that has been extensively modified to support blockface imaging. It is outfitted with a stationary Sony CCD digital video camera (as shown in Figure 4) that acquires blockface video images after each section is taken. In this system, the tissue moves horizontally across the knife, and with each stroke the knife descends the appropriate distance. The camera is located behind the knife on a mount attached to the knife housing. Thus, the focus is maintained on the blockface throughout the cutting session. Consistent repositioning of the tissue under the camera is critical. Our Cryopolycut has been customized to provide the necessary repositioning accuracy. Rather than a contact switch to turn off the forward motor at the end of the stroke, the motor output is reduced as the treadmill comes to a stop against a metal bar. This avoids a backlash.

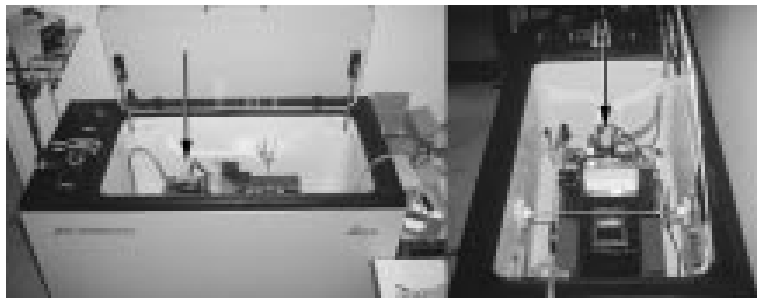

Figure 4: Shown is the cryostat used to prepare the animal tissue slices from the front and side view. Note that a CCD video camera (arrows) is located within the compartment to acquire blockface images, following each section.

\section{E. Autoradiographic Procedures}

Autoradiographic film consists of a substrate (usually gelatin or acetate) upon which an emulsion of silver halide is layered [5]. Exposure of autoradiography film is most often caused by charged particles $(\alpha, \beta-, \beta+)$. Interestingly, many radionuclides in PET and SPECT that emit high-energy $\gamma$-rays also emit short-range charged particles, and may thus be used in forming autorads. The process of autorad film exposure is illustrated in Figure 5.

\section{F. Film digitization}

The autorad sheet (an analog signal) must be converted into digital form. The routine procedure is to place the film sheet on a light box and image it with a digital camera, similar to the method of Friedman et al.[6]. Transillumination of autorads for digitization was done with a Northern Light model B95 Precision Illuminator (Imaging Research, Inc.). Stray light was minimized by masking the lightbox output to a small region the size of the autorad image. In addition, the overhead lighting was turned off. For purposes of light field homogeneity, the light box was allowed to warm up for a period of 10 minutes prior to scanning, after which time it is accepted as being stable. A CCD camera with 55mm Nikkor Micro (Nikon) lens set to f8 was used to image the film. The frame was grabbed using a Macintosh 9600 with Scion Image capture card and the image 


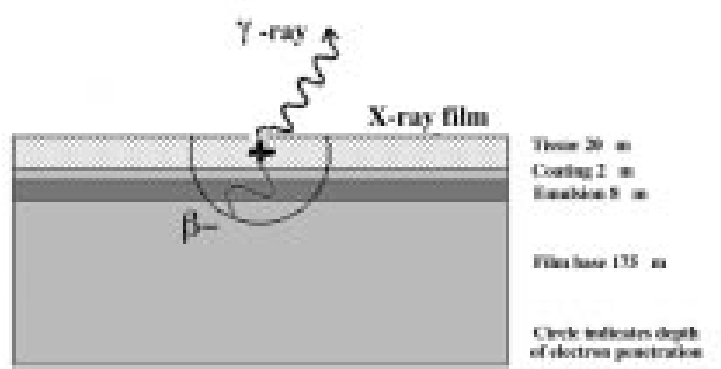

Figure 5: Illustration of the process of autorad formation. The (+) is meant to denote source location. Note that the case here is for a SPECT radiopharmaceutical, which emits a high energy $\gamma$-ray along with the electron responsible for the autorad signal. Note the intersection of the semicircle, meant to denote the radial distance traveled by the $(\beta-)$ particle, with the band corresponding to the emulsion of the film. The finite range of the $\beta$ - leads to blurring, but the degree of blur is so small as to be inconsequential.

acquisition software Brain v3.0 (Drexel University). The final pixel size of the image was $640 \times 480$.

\section{G. Activity correction}

In addition to the brain images, a film recording of radioactive standards was also digitized for the purposes of activity correction. A calibration transfer function is generated by plotting the film density [ $\left[\begin{array}{ll}0 & 255\end{array}\right]$ against radioactive concentration $(\mu \mathrm{Ci} / \mathrm{mg})$ for each of the film sheets. Each of the brain slices is transformed according to the specific film sheet calibration using a MATLAB $\mathcal{C}$ routine to represent relative radionuclide activity within the tissue.

\section{H. 2-Dimensional Image Alignment}

The alignment of the rat AR images was achieved using Brain imaging software (Drexel University). Using this software, anatomical boundaries were identified and delineated on the blockface image. The corresponding boundaries on the AR image were also delineated, and through a moments method [7], the AR is translated and rotated to match the blockface. Recall that the blockface images are in a constant reference frame, therefore alignment of the AR to the blockface on a slice by slice level achieves spatial register of the AR images throughout the volume. A blockface image is shown alongside the corresponding AR image in Figure 6.

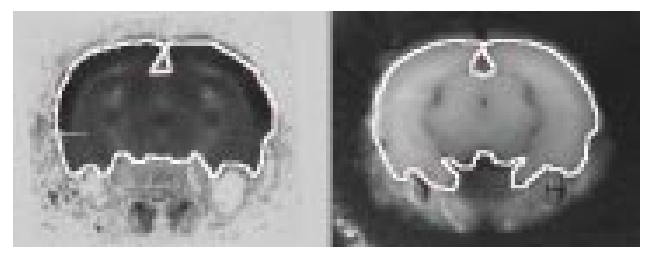

Figure 6: Video blockface image (right) was used to serially align autorad images (left) for the rat data set. The software (Brain, Drexel University) uses a moments based alignment method.

Other methods for alignment exist. For instance, one could use a fiducial landmark system, which allows for automated alignment after the data collection steps. As an example, nylon cylinders (like a rigid fishing line) could be pushed through the intact brain or the mounting block [8]. When successive slices are cut, the circular cross sections of the cylinders could be aligned manually or automatically. This method has the advantage of not requiring the user to identify what they think will be a reliable landmark for aligning. The drawback of the method is some tissue destruction if the cylinder is pushed through the tissue. Also, to be useful in autoradiography, the fiducial will need to be visualized on the film sheet.

\section{Segmentation}

The phantom may be used to evaluate reconstruction quality in terms of task-specific criteria. As an example, the quantitation of tracer uptake within specified anatomical subregions is of interest clinically and in research. As shown by Hoffman et al. [9], the accuracy of these measurements will show dependence on both the object size and shape as they relate to the imaging system point spread function, with small and / or convoluted structures being the most difficult to quantify. Phantom studies with a suitably segmented data set, as being described here, allow for the evaluation of ROI quantitation methods. Ground truth segmentation is defined here by hand-drawn anatomical boundaries imposed upon the digitized autorads. (A convenient software application for segmentation is NIH Image.) Major tissue distinctions include bone, cerebro spinal fluid, gray matter and white matter, as shown in Figure 2, panel C. Regions of the rat brain appropriate as more specific ROI's for quantitation tasks would include the hippocampus and caudoputamen, since the size of each structure being roughly $5-6 \mathrm{~mm}$ in the sagittal and coronal planes, and and $2 \mathrm{~mm}$ in the horizontal plane. The relevance of these measurements is being judged relative to the quoted resolution of current animal imaging systems being on the order of $2 \mathrm{~mm}$ [10] [11].

\section{J. 3-Dimensional Image Registration}

The blockface image data set was used as the standard to which the AR and MR were registered. The autoradiograms were individually aligned in 2-D, as described above (Section H). Co-registration of the MR images to the blockface was performed using the $3 \mathrm{D}$ rigid body alignment method in AIR 3.08 [12]. The aligned MR was resliced such that the inplane resolution matched that of the blockface $(35 \mu \mathrm{m})$, while the axial resolution matched the original MR of $1 \mathrm{~mm}$.

\section{K. Acknowledgements}

The authors would like to thank Louise Bertran, Ing-Tsung Hsiao, YuFei Huang and the University of Pittsburgh MR center. This work was supported by NIH NS32879 (Stony Brook) and NIH award P41RR01638, a Biomedical Technology Resource grant to the Computer Vision Center for Vertebrate Brain Mapping (Drexel). 


\section{REFERENCES}

[1] G. Gindi, D. Dougherty, I.T. Hsiao, and A. Rangarajan, "Autoradiographic-based phantoms for emission tomography”, In Kenneth M. Hanson, Ed., Proceedings of SPIE Medical Imaging 1997 - Image Processing, volume 3034, pp. 403-414, Newport Beach, California, February 1997, SPIE.

[2] D. Dougherty, I. Hsiao, W. Wang, and G. Gindi, "Computerized Biological Brain Phantom for Evaluation of PET and SPECT Reconstruction", IEEE Transactions in Nuclear Science, 45(3), 1998.

[3] P. Som, Y. Yonekura, Z.H. Oster, M.A. Meyer, M.L. Pelletteri, J.S. Fowler, R.R. MacGregor, J.A.G. Russell, A.P. Wolf, I. Fand, W.P. McNally, and A.B. Brill, "Quantitative Autoradiography with Radiopharmaceuticals, Part 2: Applications Radiopharmaceutical Research:Concise Communication", Journal of Nuclear Medicine, 24, pp. 238-244, 1983.

[4] C.C. Lo, J. Nissanov, and O. Tretiak, "Section Thickness Determination and Evaluation of a New Gray Value Normalization Technique for Brain Reconstruction", Technical Report 033198, Drexel University Imaging Computer Vision Center, Philadelphia, PA, 1996.

[5] J.L. Lear, R. Ackerman, M. Kameyama, R. Carson, and M. Phelps, "Multiple-Radionuclide Autoradiography in Evaluation of Cerebral Function", Journal of Cerebral Blood Flow and Metabolism, 4, pp. 264-269, 1984.

[6] H. Friedman and P. Goldman-Rakic, "Activation of the Hippocampus and Dentate Gyrus by Working-Memory: A 2-Deoxyglucose Study of Behaving Rhesus Monkeys", The Journal of Neuroscience, 8(12), pp. 4693-4706, 1988.

[7] J. Nissanov and D.L. McEachron, "Advances in image processing for autoradiography", J. Chem. Neuroanat., 4, pp. 329-342, 1991.

[8] J.L. Humm, R.M. Macklis, X.Q. Lu, Y. Yang, K. Bump, B. Beresford, and L.M. Chin, "The spatial accuracy of cellular dose estimates obtained from 3D reconstructed serial tissue autoradiographs", Phys. Med. Biol., 40, pp. 163-180, 1995.

[9] E.J. Hoffman, S.C. Huang, and M.E. Phelps, "Quantitation in Positron Emission Computed Tomography: 1. Effect of Object Size", Journal of Computer Assisted Tomography, 3(3), pp. 299-308, June 1979.

[10] S.R. Cherry, Y. Shao, S.W. Silverman, K. Meadors, S. Siegel, A. Chatziioannou, J.W. Young, W.F. Jones, J.C. Moyers, D. Newport, A. Boutenfnouchet, T.H. Farquhar, M. Andreaco, M.J Paulus, D.M. Binkley, R. Nutt, and M.E. Phelps, "MicroPET: A High Resolution PET Scanner for Imaging Small Animals", IEEE Transactions on Nuclear Science, 44(3), pp. 1161-1166, 1997.

[11] R.M. Bloomfield, S. Rajeswaran, T.J. Spinks, S.P. Hume, R. Myers, S. Ashworth, K.M. Clifford, , W.F. Jones, L.G. Byars, J. Young, M. Andreaco, C.W. Williams, A.A. Lammertsma, and T. Jones, "The design and physical characteristics of a small animal positron emission tomograph", Phys. Med. Biol., 40, pp. 1105-1126, 1995.

[12] R.P. Woods, S.T. Grafton, C.J. Holmes, S.R. Cherry, and J.C. Mazziotta, "Automated image registration: I. General methods and intrasubject, intramodality validation", Journal of Computer Assisted Tomography, 22, pp. 141154, 1998. 